\title{
Detailed Grid Integration Analysis of Distributed PV
}

\author{
Jimmy Quiroz ${ }^{1}$ and Matthew Reno ${ }^{2}$ \\ ${ }^{1}$ Sandia National Laboratories, Albuquerque, NM, 87185, USA \\ ${ }^{2}$ Georgia Institute of Technology, Atlanta, GA, 30332, USA
}

\begin{abstract}
This paper shows examples of detailed PV grid integration analysis performed by Sandia National Laboratories (SNL) on two separate distribution feeders with two different simulated PV deployments for each. Through the use of advanced modeling tools and techniques, examples of time-series detailed feeder modeling are presented. Feeders in Utah and Georgia with simulated $100 \%$ PV penetration, either central or distributed, were studied. The analysis approach of each deployment type and location on the feeder is described, as well as the use of advanced PV output estimations for modeling maximum solar variability. Comparisons of the performance measured for each feeder, including maximum steady-state voltage and voltage regulation equipment operations, are shown. Impact results from the analyses are described, as well as any potential mitigations. Future analysis aspects are discussed in relation to the detailed study findings thus far.

Index Terms - distributed power generation, photovoltaic systems, power distribution, power system interconnection, power system modeling, power system planning, power system simulation, solar power generation .
\end{abstract}

\section{INTRODUCTION}

Deployment of distribution-connected PV systems is increasing rapidly, and high-penetration scenarios have the potential to affect the operation of distribution feeders. Existing methods, data, and simulation tools may not be adequate to fully characterize system impacts. To address this problem, Sandia National laboratories (SNL) recently completed a series of in-depth studies to evaluate the potential impacts of simulated photovoltaic (PV) systems on feeders in Utah [1] and Georgia. The studies evaluated the potential impacts of adding a variety of distributed rooftop and central PV to distribution grids.

This report describes the detailed analyses of two feeders, using analysis techniques and tools that more fully characterize PV system impacts. Ultimately, the analysis methodology presented here can be extended to analyze a broad range of feeders and PV deployment scenarios to develop guidelines that can be used to assess the impact of PV on distribution circuits.

The two feeders highlighted in this report are as follows:

A) Feeder A is located in central Utah. Based on distribution transformer sizes, its load consists of approximately $70 \%$ residential customers and 30\% commercial class customers. The substation transformer nominal voltages are $46-12.47 \mathrm{kV}$, with a 9.375 MVA rating (FA). The substation transformer serves 2 feeders, with a coincident summer peak demand of
5.1 MVA during the study year. Feeder A's summer peak demand for the study year was 1.7 MVA. The longest primary conductor path from the substation is 3.9 miles. The load tap changer (LTC) is set at 122-124 volts with a 60 second time delay. The LTC is also set for line drop compensation (LDC) of $4+\mathrm{j} 3(\mathrm{R}+\mathrm{jX})$ volts at current transformer $(\mathrm{CT})$ rating. The use of LDC is explained further in Section II.B. Feeder A has two $300 \mathrm{kVAr}$ fixed capacitor banks. Feeder A does not have any line voltage regulators out on the feeder. Fig. 1 shows the topography of Feeder A.

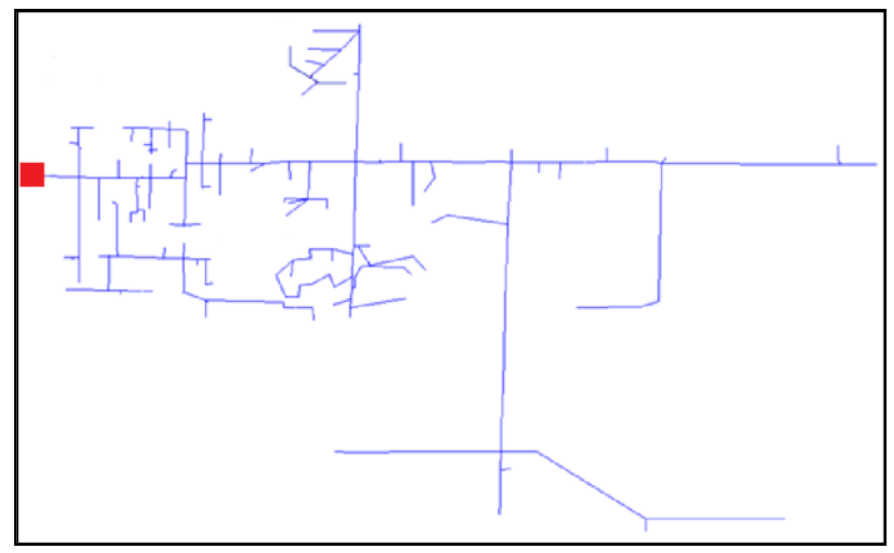

Fig. 1. Feeder A topography with the substation highlighted in red.

B) Feeder B is located in northwestern Georgia. Based on distribution transformer sizes, its load consists of approximately $40 \%$ residential customers and $60 \%$ commercial class customers. The substation transformer nominal voltages are $115-20 \mathrm{kV}$, with a 40 MVA rating (FA). The substation transformer serves 5 feeders, with a coincident summer peak demand of 31.9 MVA during the study year. Feeder B's summer peak demand for the study year was 7.5 MVA. The longest primary conductor path from the substation is 3.4 miles. The load tap changer (LTC) is set at 119-122 volts with a 30 second time delay. The LTC is also set for LDC of $6+\mathrm{j} 2$ volts. Feeder $\mathrm{B}$ has one $900 \mathrm{kVAr}$ fixed capacitor bank and two $1200 \mathrm{kVAr}$ switching capacitor banks. The switching capacitor banks are set for voltage control with an ON setting of 121 volts and an OFF setting of 123.5 volts, with a 30 second time delay. Feeder B does not have any line voltage regulators out on the feeder. Fig. 2 shows the topography of Feeder B. 


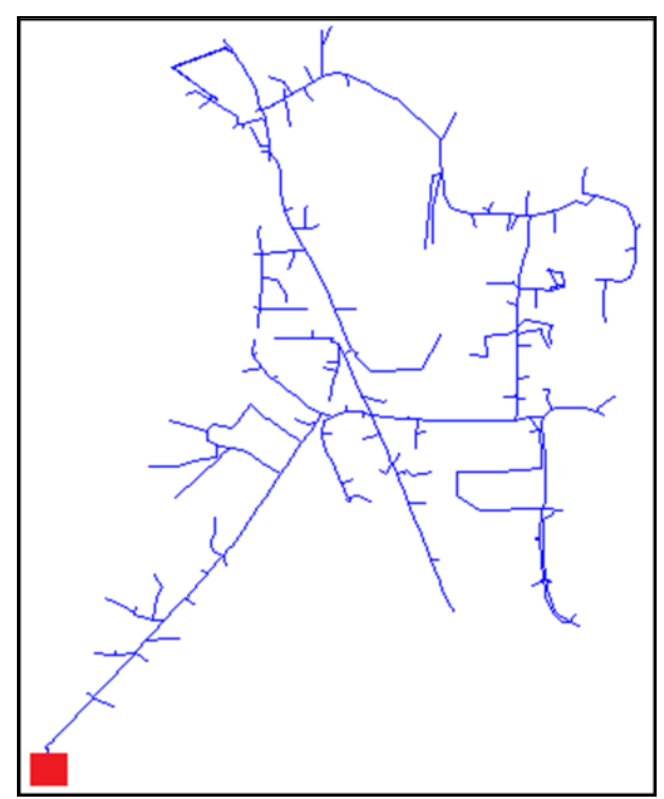

Fig. 2. Feeder B topography with the substation highlighted in red.

\section{ANALYSIS METHODOLOGY}

For each feeder, two PV deployment scenarios were investigated, including both central and distributed rooftop $\mathrm{PV}$, at $100 \%$ penetration levels, where the rated PV capacity was equal to the annual feeder peak load. The Open Distribution System Simulator ${ }^{\mathrm{TM}}$ (OpenDSS) was used to perform the electrical power flow studies.

\section{A. Time-Series Modeling}

OpenDSS was developed by the Electric Power Research Institute (EPRI). One of the main reasons for using OpenDSS, as opposed to industry-standard distribution analysis software, is the ability to conduct high-resolution time series analysis. Currently available utility-standard simulation tools are not generally well suited for sequential or extended dynamic simulations needed to fully characterize the effects of PV output variability on distribution feeders.

To conduct the studies, existing feeder models were converted to OpenDSS format. The working cases converted to OpenDSS format were validated by comparing power flow results against results obtained with commercial software; under the same load conditions. OpenDSS is very flexible with respect to scenario analysis; however, it has a basic interface that supports a manual, script-based study process. To facilitate the analysis in OpenDSS, an interface was developed using MATLAB. This enabled the customization of results display and saving of data. The use of a MATLAB interface also enabled the ability to integrate solar data inputs [2]. Section II.E provides additional details on solar data estimation.

The source impedances for the substations, i.e., the high side of the substation transformers, were modeled according to short-circuit data provided for each. The coincident demand of the other feeder(s) served by the substation transformers were modeled as an aggregate lumped load at the substation based on measured total feeder load data provided for each.

\section{B. Impact Analysis}

The specific technical impacts analyzed in the electrical study were maximum steady state voltages and voltage regulation equipment operations.

The voltage ranges defined by the ANSI C84.1 standard were used as guidelines for acceptable voltage levels. Fig. 3 shows the ANSI voltage ranges for service voltage, which is defined as the point of common coupling between customer and utility. To obtain the maximum steady state voltage, the maximum voltage found anywhere on the feeder model during the entire simulation period was identified for each case. For Feeder A, the maximum voltages correspond to the highest voltages found on the primary system, any voltage drop or rise on the secondary system is not captured. For Feeder B, which had representations of the secondary system modeled, voltages may correspond to the secondary system, wherever appropriate.

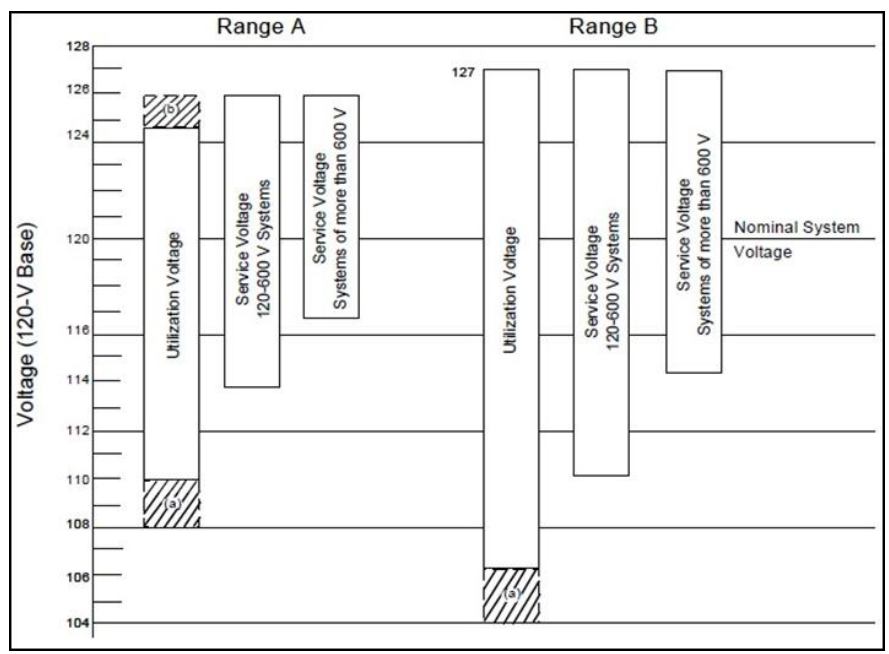

Fig. 3. ANSI C84.1 Range A and B service voltage limits [3].

For the voltage regulation equipment operation analysis, the activity of the load tap changer (LTC) of each substation transformer was monitored and quantified for comparison to the base case without PV. For Feeder B, the operations of the switching capacitors were also monitored. Neither feeder had line voltage regulators to monitor.

The LTC is a voltage regulation device connected to the secondary side of the substation transformer. The LTC has the ability to buck or boost the distribution voltage level $\pm 10 \%$ through a series of steps, typically 16 in each direction, with a neutral position. For Feeder B, the LTC is set for \pm 8 steps. The basic settings on a LTC include a voltage level and 
bandwidth, as well as a time delay setting (typically 30-90 seconds). The sequential mode of control, the most commonly implemented, was simulated in this study. In sequential mode the LTC continuously monitors the voltage level. When an out of band voltage is detected, the time delay timer is initiated. If the voltage deviation remains out of band for the duration of the time delay, a tap change, or series of tap changes, is executed until the voltage returns to within the band. If the voltage deviation returns to within band before the timeout of the time delay, the timer is reset. For this reason, one-second resolution was chosen for the time series data inputs.

Both feeder LTC settings also utilized the line drop compensation (LDC) feature. LDC is used to assign the desired voltage control at a remote point from the location of the LTC. This requires the specification of the estimated voltage drop ( $\mathrm{R}$ and $\mathrm{X}$ ) of the line segment between the LTC location and the desired control point, at CT-rated current. The LDC feature is current dependent, estimating the voltage drop across the specified impedance, and operating the LTC accordingly. LDC is especially useful for line voltage regulators, where the optimal location of voltage regulation on the feeder may present physical installation challenges and the regulator bank can be installed at a more ideal location nearby, while still achieving the desired voltage regulation.

\section{PV Deployment Scenarios}

Two PV deployment scenarios were modeled on each feeder, as well as a base case with no PV for comparison. Each deployment scenario represented a penetration level of $100 \%$ of each feeder's MVA peak load. The nominal PV system outputs for Feeder A and Feeder B were 1.7 MW and 7.5 MW, respectively. All PV systems were assumed to be unity power factor output plants, producing only real power (watts).

Two different deployment types were simulated on each feeder: one central PV system case and one distributed rooftop PV case. The central case was simulated as a dedicated single PV plant with high PV density (i.e., high ground coverage ratio) connected to a single point of common coupling (PCC) on the distribution system. The distributed rooftop PV case consisted of several smaller systems distributed across a larger area of residential and commercial customers, resulting in a lower PV density spanning a larger geographical area. Both the central and distributed cases contained the same amount of total PV.

The central PV system was connected at the 3-phase bus with the highest impedance from the substation that could thermally accommodate the system size. The distributed rooftop area was chosen near the middle region of the feeder containing a mix of all three phases of customers. The distributed rooftop PV systems were connected to the primary bus of the distribution transformers.

\section{Load Data Inputs}

The time period studied for each feeder was chosen based on when PV output could be largest relative to load. Each feeder's most recent annual load data was compared to coincident local clear-sky irradiance values and the highest irradiance-to-load ratio was identified. Clear-sky irradiance was used on the assumption that the higher the clear-sky irradiance, the higher the PV output. The periods of maximum irradiance-to-load ratio do not necessarily correspond to the absolute minimum load on the feeders or the periods of maximum possible $\mathrm{PV}$ output, but rather represent when the $\mathrm{PV}$ is suspected to have the greatest effect on the impacts studied. The peak penetration points found for Feeder A and Feeder B were Sunday, June 13, 2010 at 12:45 PM MST, and Saturday, April 23, 2011 at 12:00 PM EST, respectively.

To incorporate day-of-the-week load diversity, an entire week surrounding the peak penetration point day was simulated. Considering the high resolution of the simulation, longer periods than a week would be more time consuming and data intensive. As is customary, one-second resolution load data was not available for the feeders, so the available 15minute data for Feeder A and 1-hour load data for Feeder B were interpolated to 1-second resolution. The addition of noise to simulate load variability at one-second resolution was not attempted since this would require extensive regional, seasonal, and customer class analysis to develop a technically feasible approach. As can be seen in Fig. 4, there is a significant difference in load profile between weekdays and weekends.

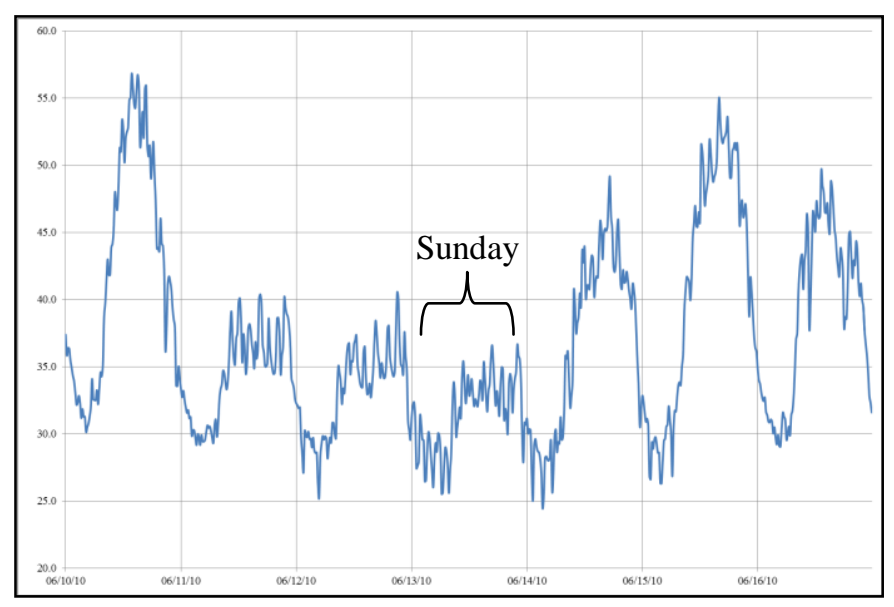

Fig. 4. Load shape for Feeder A during the study week.

The total feeder load was allocated to each individual load modeled along the entire feeder based on connected kVA transformer sizes. Based on the measured data, a power factor of 0.90 lagging was assumed for each load. For Feeder A, balanced three-phase load conditions were assumed based on the actual three-phase average load data. For Feeder B, load measurements for each phase were used, reflecting measured 
phase unbalance. For Feeder A, the loads were modeled on the primary system. For Feeder B, which had representations of the secondary system modeled, loads were modeled on the secondary system.

\section{E. PV System Data Inputs}

High resolution (one-second) solar input data is critical to run the simulations, but availability of such data can be problematic. If the solar data used is not representative of the local climate, then the study results may not be useful. When it is not possible to obtain local PV power plant output for the specific deployment scenario of interest, estimated PV power output profiles need to be developed using measured or statistically-representative surrogate irradiance data.

Locally measured high resolution irradiance was unavailable for Feeder A, so surrogate data was used. Measured solar irradiance data from a network in Colorado was the most feasible data available. For Feeder B, measured irradiance data from seven irradiance sensors installed on the feeder were used to develop PV output data sets for analysis.

The Wavelet-based Variability (WVM) was used to estimate PV power output using the irradiance data (surrogate or measured). The WVM is a method for estimating PV power plant output, given measurements from an irradiance point sensor, by determining the geographic smoothing that will occur over the area of the plant [4]. Different amounts of smoothing are applied at different timescales by using the wavelet transform.

For each feeder, a highly-variable day was chosen by using a variability index (essentially mean ramp rates) as a metric of variability. The high variability day identified for each location was then duplicated for the entire study week to simulate a worst case assumption that every day in the study week could be highly-variable. A shortcoming of this method is the elimination of the correlation between PV system output and load demand under similar atmospheric conditions. However, this method was the most technically feasible way to provide a worst-case scenario for variability impacts.

Since the high variability day did not coincide with the study week dates, and in the case of Feeder A the data was from a different location, the irradiance data was scaled and shifted to correspond to the correct length of day, sunrise and sunset times, and intensity of solar radiation. This was done by picking a section of the clear-sky index of the source data to repeat. After this extension, the clear-sky index was multiplied by the feeder local clear-sky model and adjusted to start at the local sunrise time, creating representative point sensor irradiance data for each feeder. Fig. 5 shows a visual representation of this transformation.

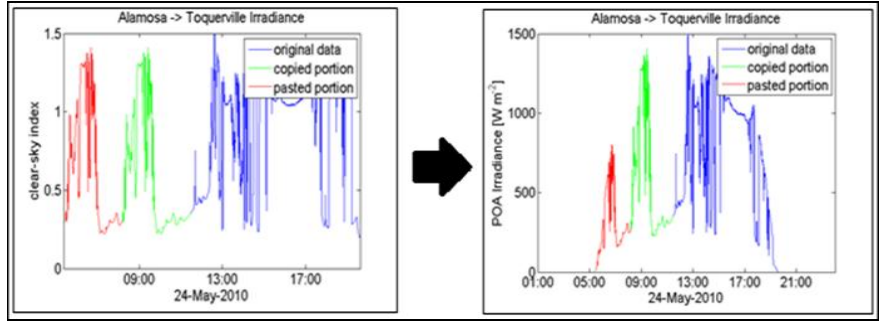

Fig. 5. PV output synthesis for sunrise/sunset synchronization

\section{IMPACT RESULTS}

Table I shows the results of the analysis on each feeder for the central PV case. Both the maximum voltages ( $120 \mathrm{~V}$ base) and LTC operation counts are shown, including the base case results for comparison. Voltage levels above ANSI Range A are highlighted with red font. The change in LTC operations from the base case is shown in parenthesis.

\begin{tabular}{|c|c|c|c|c|}
\hline \multicolumn{3}{|c|}{ Table I: Central PV system, $\mathbf{1 0 0 \%}$ penetration by feeder peak } \\
load, located at end of feeder. \\
\hline \multirow{2}{*}{ Feeder } & \multicolumn{2}{|c|}{ Maximum Voltage } & \multicolumn{2}{|c|}{ LTC Operations } \\
\cline { 2 - 5 } & $\begin{array}{l}\text { Base } \\
\text { Case }\end{array}$ & With PV & $\begin{array}{c}\text { Base } \\
\text { Case }\end{array}$ & With PV \\
\hline Feeder A & 124.9 & 129.6 & 10 & $8(-2)$ \\
\hline Feeder B & 125.3 & 125.9 & 9 & $13(+4)$ \\
\hline
\end{tabular}

Table II shows the results measured for each feeder under the distributed rooftop PV scenario.

\begin{tabular}{|c|c|c|c|c|}
\hline \multicolumn{4}{|c|}{ Table II: Distributed rooftop PV systems, $\begin{array}{c}\mathbf{1 0 0} \% \text { penetration by } \\
\text { feeder peak load, middle of feeder. }\end{array}$} \\
\hline \multirow{3}{*}{ Feeder } & \multicolumn{2}{|c|}{ Maximum Voltage } & \multicolumn{2}{|c|}{ LTC Operations } \\
\cline { 2 - 5 } & $\begin{array}{l}\text { Base } \\
\text { Case }\end{array}$ & With PV & $\begin{array}{c}\text { Base } \\
\text { Case }\end{array}$ & With PV \\
\hline Feeder A & 124.9 & 127.9 & 10 & $6(-4)$ \\
\hline Feeder B & 125.3 & 125.3 & 9 & $13(+4)$ \\
\hline
\end{tabular}

For the high voltages found on Feeder A under the end-offeeder central PV case and the mid-feeder distributed case, there are a few things to consider. First, it is somewhat rare to find a feeder with such a high setting and line drop compensation. The LDC settings as defined here, attempt to regulate the voltage at a point on the feeder beyond the actual location of the LTC. LDC settings are defined based on real and reactive impedance, so the LTC adjusts to the load current in order to maintain the regulation range at the defined point. This is complicated when more than one feeder is being served by the substation LTC. The LDC settings will translate to different locations on each feeder. The choice of LTC settings is usually intended to maximize the cycling of the device through the neutral step, which is directly dependent on the transmission source impedance, transmission source voltage, 
transformer high side no-load voltage tap, transformer impedance, and transformer loading.

The LTC operation impacts observed were minimal. For Feeder A, a slight decrease in operations was observed. This is due to the offset of load and voltage rise that results from the integration of PV. The voltage increase may eliminate the out of band low voltages during the day.

Table III shows the operations summary for the active switching capacitor bank on Feeder B.

\begin{tabular}{|c|c|}
\hline \multicolumn{2}{|c|}{ Table III: Feeder B switching capacitor bank operations. } \\
\hline Scenario & Number of Operations \\
\hline Base Case & 11 \\
\hline Central PV, End of Feeder & 3 \\
\hline Distributed Rooftop PV, Middle of Feeder & 3 \\
\hline
\end{tabular}

A similar decrease in operations on the switching capacitor bank on Feeder B was observed. Only one of them had any switching activity due to the light loading of the study period. The active bank showed a measurable decrease in operations from the base case to the PV integration cases. The switching capacitor banks are set for voltage support, with low limit and high limit voltage settings that control when to switch the banks on and off, respectively. The presence of PV and the resulting voltage rise eliminates some of the instances where voltage support was needed in the base case.

\section{CONCLUSIONS AND DISCUSSION}

For mitigating the high voltages found, it would be necessary to consider several factors. One consideration would be the PF output of the plant. Many inverters are now capable of sourcing and/or absorbing reactive power, which can reduce the voltage impact of the system. Another consideration is the use of LDC. Reducing the settings or eliminating the LDC in these cases could be investigated as a mitigation technique, or a re-evaluation of the LTC voltage setting to a lower setting could be explored.

The LTC operation impacts observed, despite the high variability PV outputs used and the high penetration levels, were minimal. In other studies, SNL has found significant increases in LTC operations during a study week for some cases. In future studies, it would be more valuable to investigate an annual quantification using coincident and local irradiance to capture the correlation of load and PV behavior under similar atmospheric conditions, though this is very data and time intensive.

The relationship found between penetration level and grid impact is a significant result. It demonstrates that in some cases the amount of PV penetration that can be accommodated without adverse grid impacts studied is far in excess of $15 \%$, closer to the $100 \%$ used here. The results also show that high penetration scenarios show clear evidence that grid impacts do occur for some circuits but not for others. Understanding what factors cause the variety of impacts and relating these factors to fundamental characteristics of the feeder topology and operation will be a valuable area for future research.

Time series analysis capability proves to be essential in properly analyzing these impacts of PV integration. The incorporation of LTC time delay and the corresponding voltage levels on the feeder would be nearly impossible to duplicate using snap-shot analysis tools commonly used for integration studies. Analysis has shown that, in many cases, the highest voltage found on a feeder during a study week does not always occur during the point of highest PV penetration, and it is therefore necessary to capture the interaction with the voltage regulation settings. Time series analysis is also necessary for quantifying impacts, e.g. the duration of a voltage deviation or the number of LTC operations during a period.

The present state of analysis tools results in a time and data intensive process for developing time series analysis capability. Given the many different software tools in use, as well as variations in the way different customers use the same software, it would be impossible to design a single tool to convert models into time series capable platforms, such as OpenDSS. The running time and data management of long term, high resolution power flows is also very challenging.

Future research in detailed modeling will continue to unveil the capabilities needed to properly analyze integration impacts, including others not studied here, and recommend best practices for integration studies and improve interconnection screens. This would require analysis on a larger and more diverse set of feeders, maximizing the relevance of findings.

\section{ACKNOWLEDGEMENT}

Sandia National Laboratories is a multi-program laboratory managed and operated by Sandia Corporation, a wholly owned subsidiary of Lockheed Martin Corporation, for the U.S. Department of Energy's National Nuclear Security Administration under contract DE-AC04-94AL85000.

Portions of this study were performed in collaboration with the Electric Power Research Institute (EPRI), as part of a Cooperative Research and Development Agreement (CRADA). 


\section{REFERENCES}

[1] J. Quiroz and C. Cameron, "Technical Analysis of Prospective Photovoltaic Systems in Utah," Sandia Report SAND 20121366, 2012.

[2] M. J. Reno, A. Ellis, J. Quiroz, and. S. Grijalva, "Modeling Distribution System Impacts of Solar Variability and Interconnection Location," World Renewable Energy Forum, 2012.

[3] ANSI C84.1-1995, Electrical Power Systems and Equipment Voltage Ratings (60 Hz), 1995, American National Standards Institute, 25 West $43^{\text {rd }}$ Street, $4^{\text {th }}$ Floor, New York, NY 10036, USA.

[4] M. Lave and J. Kleissl, "Testing a Wavelet-based Variability Model (WVM) for Solar PV Powerplants," 2012 IEEE Power \& Energy Society General Meeting, 2012. 